\title{
Biological treatment provides disease-modifying immunological effects
}

\author{
Mohamed Shamji ${ }^{1}$, Elizabeth Palmer ${ }^{1}$, Janice Layhadi ${ }^{1}$, Theo Moraes ${ }^{2}$, and Thomas \\ Eiwegger ${ }^{3}$ \\ ${ }^{1}$ Imperial College London \\ ${ }^{2}$ University of Toronto \\ ${ }^{3}$ The Hospital for Sick Children
}

April 3, 2021

\begin{abstract}
Advances in molecular biology alongside the accelerated development of gene and cell engineering have contributed to the development of several endotype-targeted biological therapies against chronic immune-mediated allergic diseases. Conventional therapies for asthma, chronic rhinosinusitis with polyposis (CRSwNP), chronic spontaneous urticaria and atopic dermatitis (AD) are not without limitations, and as such the advent of biological therapies have provided a promising alternative treatment option. Biologicals have proven efficacious in the treatment of refractory chronic spontaneous urticaria, asthma, AD, CRSwNP and there is increasing evidence for their utility in treating food allergy. Biologicals are applied and investigated for the most urgent need: acute treatment, symptom control and reduction of steroid usage. Currently there are five approved biologicals for allergic disease management, targeted against IgE (omalizumab), type 2 (T2) cytokines and cytokine receptors (IL-4Ra; dupilumab, IL-5; mepolizumab/reslizumab, IL-5Ra; benralizumab).
\end{abstract}

\section{Biological treatment provides disease-modifying immunological effects}

Mohamed H Shamji ${ }^{1}$, Elizabeth Palmer ${ }^{1}$, Janice A. Layhadi ${ }^{1}$, Theo J Moraes ${ }^{2}$, Thomas Eiwegger ${ }^{3,4,5}$

${ }^{1}$ Immunomodulation and Tolerance Group, Allergy and Clinical Immunology, Inflammation, Repair and Development, National Heart and Lung Institute, Imperial College London. MRC \& Asthma UK Centre in Allergic Mechanisms of Asthma, London, United Kingdom

${ }^{2}$ Division of Respiratory Medicine, Departments of Paediatrics, The Hospital for Sick Children, University of Toronto, Toronto, ON, Canada

${ }^{3}$ Translational Medicine Program, Research Institute, Hospital for Sick Children, Toronto, Ontario, Canada

${ }^{4}$ Division of Immunology and Allergy, Food Allergy and Anaphylaxis Program, The Department of Pediatrics, Hospital for Sick Children, Toronto, Ontario, Canada

${ }^{5}$ Department of Immunology, Faculty of Medicine, University of Toronto, Toronto, Ontario, Canada

\section{Corresponding authors:}

Mohamed H. Shamji, PhD

Immunomodulation and Tolerance Group, Allergy \& Clinical Immunology

Inflammation, Repair and Development, National Heart \& Lung Institute, Imperial College London, $1^{\text {st }}$ Floor, Room 111, Sir Alexander Fleming Building, South Kensington Campus 
London SW7 2AZ, United Kingdom

Tel: +44 (0) 2075941673 , Mobile: +44 (0) 7872850369 .

Emails: m.shamji@imperial.ac.uk

Thomas Eiwegger, MD

Division of Immunology and Allergy, Food Allergy and Anaphylaxis Program, The Department of Paediatrics, Hospital for Sick Children, 555 University Ave, ON, Toronto, Canada,

E-mail: thomas.eiwegger@sickkids.ca

Tel.: +1 416-813-7654 ext. 1862

\section{INTRODUCTION}

Advances in molecular biology alongside the accelerated development of gene and cell engineering have contributed to the development of several endotype-targeted biological therapies against chronic immunemediated allergic diseases. Conventional therapies for asthma, chronic rhinosinusitis with polyposis (CRSwNP), chronic spontaneous urticaria and atopic dermatitis (AD) are not without limitations, and as such the advent of biological therapies have provided a promising alternative treatment option. Biologicals have proven efficacious in the treatment of refractory chronic spontaneous urticaria, asthma, AD, CRSwNP and there is increasing evidence for their utility in treating food allergy ${ }^{1-3}$. Biologicals are applied and investigated for the most urgent need: acute treatment, symptom control and reduction of steroid usage. Currently there are five approved biologicals for allergic disease management, targeted against IgE (omalizumab), type 2 (T2) cytokines and cytokine receptors (IL- $4 \mathrm{R} \alpha \cdot$ dupilumab, IL-5; mepolizumab/reslizumab, IL-5R $\alpha \cdot$ benralizumab) $)^{2}$.

\section{PROS: BIOLOGICALS ARE SAFE AND EFFECTIVE IN TREATING ALLERGIC DISEASES}

- Omalizumab is an anti-IgE humanized IgG1 monoclonal antibody (mAb) which forms complexes with soluble IgE, inhibiting binding to its high-affinity IgE-receptor (FceRI) on effector cells such as basophils, eosinophils and mast cells ${ }^{1}$. The paucity of IgE-mediated cross-linking of allergen reduces effector cell activation and the subsequent release of immune mediators such as histamine, responsible for driving the salient symptoms of atopic disease. Moreover, through reducing serum-free IgE, omalizumab downregulates FceRI expression on mast cells, basophils and dendritic cells and IgE-CD23 interactions on B-cells ${ }^{1}$, in turn downregulating IgE synthesis and T helper 2 (Th2) cell differentiation.

- Data from two phase III clinical trials demonstrates that omalizumab reduces disease exacerbations by $\sim 55 \%$ in severe asthma patients ${ }^{4}$ and leads to significant improvements in mean nasal polyp scores (NPS) and nasal congestion scores (NCS) in CRSwNP patients compared to placebo ${ }^{5}$. Moreover, omalizumab significantly improves patient quality of life compared to conventional therapies in severe asthma $^{6}, \mathrm{CRSwNP}^{5}$ and chronic spontaneous urticaria ${ }^{7}$ for which it is authorized.

- Mepolizumab and reslizumab are humanized IgG1 and IgG4 mAbs respectively which bind directly to IL-5, inhibiting the activation and recruitment of eosinophils ${ }^{1}$. Benralizumab, another humanized $\mathrm{IgG}_{1} \mathrm{mAb}$, targets the IL-5 pathway through binding to the $\alpha$ subunit of the IL-5R, impeding IL-5 signal transduction ${ }^{1}$. Moreover, benralizumab binds to Fc $\gamma$ RIIIa on natural killer cells through its Fc region, depleting eosinophils through antibody-dependent cellular cytotoxicity ${ }^{1}$.

- Anti-IL-5 biologics demonstrate the most efficacy against allergies characterized by eosinophilia, such as severe eosinophilic asthma and CRSwNP. Systematic reviews have demonstrated that mepolizumab, reslizumab and benralizumab reduce asthma incidence rate ratios by $0.49,0.46$ and 0.53 , respectively and report that mepolizumab and benralizumab reduce required daily oral corticosteroid dose ${ }^{6}$. Moreover, preliminary data from phase III trials have reported that severe CRSwNP patients treated with mepolizumab (SYNAPSE: NCT03085797) or benralizumab (OSTRO: NCT03401229) as an add-on therapy demonstrate improvements in total endoscopic NPS and sinonasal symptoms, supporting their regulatory body approval for disease management in the future. 
- Dupilumab is a human IL-4R $\alpha$ blocking IgG4 mAb which inhibits the sister cytokines IL-4/IL-13 through blockade of their shared receptor subunit ${ }^{1}$. IL-4 plays a key role in T2 immunity by promoting Th2 differentiation and IgE class-switching in B cells.

- Originally approved for the treatment of $\mathrm{AD}$, dupilumab significantly improved symptom scores including Eczema Area Severity Index and itch Numeric Rating Scale scores during phase III clinicals ${ }^{8}$. Dupilumab is also authorised for the treatment of severe asthma ${ }^{1,6}$ and severe CRSwNP ${ }^{9}$.

- Therefore, T2-targeting biologicals have greatly improved treatment efficacy compared to conventional therapies in asthma, CRSwNP, chronic spontaneous urticaria and AD.

- Novel biologicals are in development at a rapid pace, including epithelial and keratinocytes-derived mediators and cytokines such as thymic stromal lymphopoietic, IL-31 and IL-33, and may provide further improvements in the management of T2-mediated diseases in the future.

CONS: BIOLOGICALS DO NOT PREVENT OR MODIFY PROGRESSION OF ALLERGIC DISEASES UPON DISCONTINUATION

A positive disease-modifying immunological effect would indicate that biologicals prevent the onset or the progression of the disease, the development of associated co-morbidities, support tolerance development by allergen immunotherapy (AIT) or create a persistent beneficial effect after cessation of the treatment.

- Few datasets address the issue of revision or sustainability of a biological-related remission upon discontinuation. Discontinuation of dupilumab resulted in a risk of re-initiation of AD in almost $80 \%$ of patients. ${ }^{10}$ Omalizumab cessation after one year of asthma treatment led to $68 \%$ relapse rate in omalizumab responders. ${ }^{11}$ For mepolizumab, one randomized, placebo-controlled trial on subcutaneous mepolizumab against placebo after long term mepolizumab treatment in asthmatics (COMET trial) described a $59 \%$ asthma relapse and a 4-5 fold increase in blood eosinophil counts. ${ }^{12}$

- Few datasets on synergies of biologicals with AIT have been reported. Omalizumab in addition to peanut, milk, multi-food or hymenoptera immunotherapy significantly reduced the time to maintenance dose and the rate of side effects. However, it does not enhance long term clinical outcomes or increases the rate of tolerance or sustained unresponsiveness of food AIT. ${ }^{13}$

- Thus, novel approaches to mediate the dose reduction process in omalizumab facilitated OIT are needed. ${ }^{14}, 15$ One double-blind placebo-controlled study investigated a non-marketed anti-IL-4 antibody (VAK694) in the context of a suboptimal subcutaneous grass pollen AIT. Despite some promising mechanistic findings, no additional clinical benefit to the AIT was observed.

- Dupilumab, approved for T2 asthma, CRSwNP and AD addresses both the IL-4 and IL-13 axis, thus potentially affecting disease progression through interfering with isotype switching and IL-13 dependent T-follicular helper cell function. However, human data from controlled trials are missing.

- Once omalizumab therapy is ceased if used as a monotherapy for food allergy, symptoms re-occur.

- Eosinophilia or eosinophil infiltration of the lung or the GI-tract is a common phenomenon observed in patients with atopic diseases. Anti-IL5 or IL-5R targeting antibodies could have synergistic effects by abolishing this eosinophil driven inflammation on the one hand and increase safety by controlling asthma during AIT. No controlled trials are available until now.

- Recently increased infectious events have been reported for benralizumab in severe asthma patients, which has been linked to the presence of auto-antibodies. ${ }^{16}$ This is very interesting as this signal was absent in phase III benralizumab trials and require further consideration for future applications in vulnerable populations.

- Even less clinical evidence is available for a preventive or disease-modifying effect of biologicals. It is tempting to speculate on disease-modifying or preventive effects of biologicals addressing IL-4/IL-13 pathway or upstream like anti-IL-33 on very young children or even in pregnancy. However, more safety data from controlled trials are required before approaches interfering with immune pathways in childhood and very early in life ( $<2$ years) can be considered in such vulnerable populations.

In conclusion, there is no evidence that biologicals can sustainably break vicious cycles of inflammation and thereby rescind or stop the progression of the disease. Moreover, we do not have sufficient evidence to suggest 
a role of $\mathrm{T} 2$ immunity targeting biologicals to promote tolerance development to allergens when applied with or without AIT.

\section{References:}

1. Agache I, Akdis C, Akdis M, Canonica GW, Casale T, Chivato T, et al. EAACI Biologicals GuidelinesRecommendations for severe asthma. Allergy 2020.

2. Breiteneder H, Diamant Z, Eiwegger T, Fokkens WJ, Traidl-Hoffmann C, Nadeau K, et al. Future research trends in understanding the mechanisms underlying allergic diseases for improved patient care. Allergy 2019; 74:2293-311.

3. Eiwegger T, Hung L, San Diego KE, O'Mahony L, Upton J. Recent developments and highlights in food allergy. Allergy 2019; 74:2355-67.

4. Casale TB, Chipps BE, Rosén K, Trzaskoma B, Haselkorn T, Omachi TA, et al. Response to omalizumab using patient enrichment criteria from trials of novel biologics in asthma. Allergy 2018; 73:490-7.

5. Gevaert P, Omachi TA, Corren J, Mullol J, Han J, Lee SE, et al. Efficacy and safety of omalizumab in nasal polyposis: 2 randomized phase 3 trials. J Allergy Clin Immunol 2020; 146:595-605.

6. Agache I, Beltran J, Akdis C, Akdis M, Canelo-Aybar C, Canonica GW, et al. Efficacy and safety of treatment with biologicals (benralizumab, dupilumab, mepolizumab, omalizumab and reslizumab) for severe eosinophilic asthma. A systematic review for the EAACI Guidelines - recommendations on the use of biologicals in severe asthma. Allergy 2020; 75:1023-42.

7. Zuberbier T, Aberer W, Asero R, Bindslev-Jensen C, Brzoza Z, Canonica GW, et al. The EAACI/GA(2) LEN/EDF/WAO Guideline for the definition, classification, diagnosis, and management of urticaria: the 2013 revision and update. Allergy 2014; 69:868-87.

8. Gooderham MJ, Hong HC, Eshtiaghi P, Papp KA. Dupilumab: A review of its use in the treatment of atopic dermatitis. J Am Acad Dermatol 2018; 78:S28-S36.

9. Bachert C, Han JK, Desrosiers M, Hellings PW, Amin N, Lee SE, et al. Efficacy and safety of dupilumab in patients with severe chronic rhinosinusitis with nasal polyps (LIBERTY NP SINUS-24 and LIBERTY NP SINUS-52): results from two multicentre, randomised, double-blind, placebo-controlled, parallel-group phase 3 trials. Lancet 2019; 394:1638-50.

10. Silverberg JI, Guttman-Yassky E, Gadkari A, Kuznik A, Mallya UG, Mastey V, et al. Real-world persistence with dupilumab among adults with atopic dermatitis. Ann Allergy Asthma Immunol 2020.

11. Molimard M, Mala L, Bourdeix I, Le Gros V. Observational study in severe asthmatic patients after discontinuation of omalizumab for good asthma control. Respir Med 2014; 108:571-6.

12. Moore WC, Kornmann O, Humbert, Poirier MC, Bel EH, Kaneko N, et al. Outcomes Following Continuation or Stopping Long-Term Mepolizumab Treatment in Patients with Severe Eosinophilic Asthma: The Randomized Comet Trial. Am J Respir Crit Care 2020; 201.

13. MacGinnitie AJ, Rachid R, Gragg H, Little SV, Lakin P, Cianferoni A, et al. Omalizumab facilitates rapid oral desensitization for peanut allergy. J Allergy Clin Immunol 2017; 139:873-81 e8.

14. Azzano P, Paquin M, Langlois A, Morin C, Parizeault G, Lacombe-Barrios J, et al. Determinants of omalizumab dose-related efficacy in oral immunotherapy: evidence from a cohort of 181 patients. J Allergy Clin Immunol 2020.

15. Langlois A, Lavergne MH, Leroux H, Killer K, Azzano P, Paradis L, et al. Correction to: Protocol for a double-blind, randomized controlled trial on the dose-related efficacy of omalizumab in multi-food oral immunotherapy. Allergy Asthma Clin Immunol 2020; 16:38. 
16. Poznanski SM, Mukherjee M, Zhao N, Huang C, Radford K, Ashkar AA, et al. Asthma exacerbations on benralizumab are largely non-eosinophilic. Allergy 2020.

Figure 1. Legend.

Mechanisms of action of type-2 immunity targeting biologicals . 1. Omalizumab binds to soluble IgE released from B-cells, reducing the quantity of free IgE available to bind to FceRI on basophils, mast cells and eosinophils, reducing IgE cross-linking. A reduction of free soluble $\operatorname{IgE}$ also downregulates FceRI expression on the surface on the surface of effector cells. Moreover, the paucity of available IgE reduces IgE-CD23 interactions on B-cells, reducing Th2 differentiation and IgE synthesis. Omalizumab is approved for use in allergic diseases of the airways (asthma), nasal cavity (chronic rhinosinutis with nasal polyps) and skin (spontaneous urticaria). 2. Mepolizumab and reslizumab bind to IL-5 released from Th2 and ILC2 cells, inhibiting its ability to interact with IL-5R on eosinophils. Benralizumab binds to the IL-5R $\alpha$ subunit via its Fab region, similarly preventing IL-5 interaction. Benralizumab also binds to the FcrRIIIa subunit on NK cells via its Fc region, initiating ADCC through perforin and granzyme B secretion. Mepolizumab, reslizumab and benralizumab are approved for use in the airways (asthma) and mepolizumab is under approval review from the FDA for the treatment of chronic rhinosinutis with nasal polyps. 3. Dupilumab inhibits IL-4 and IL-13 released from Th2 and ILC2 cells from interacting with their shared IL-4R $\alpha$ subunit. This in turn prevents IL-4-mediated stimulation of soluble IgE synthesis from B-cells and activation of basophils, reducing their associated immune mediator release. Inhibition of IL-13 reduces goblet cell hypertrophy and mucus production. Dupilumab is approved for use in the airways (asthma), nasal cavity (chronic rhinosinutis with

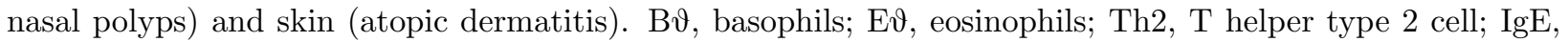
immunoglobulin E; ILC2, type 2 innate lymphoid cell; IL-5R, IL-5 receptor; IL-4R, IL-4 receptor; IL-13R, IL13 receptor; FceRI, high affinity IgE receptor; Fc $r$ RIIIa, low low-affinity Fc receptor for immune-complexed IgG; NK, natural killer cells; ADCC, antibody-dependent cellular cytotoxicity. 